\title{
A Symmetric Searchable Encryption Identification of Data on Probabilistic Trapdoors
}

\author{
N. V. V. Satyanarayana, J. M. S. V. Ravi Kumar, M. Babu Reddy, N. Leelavathi, B. Sujatha
}

\begin{abstract}
Accessible Encryption (SE) permits a client in accordance with transfer records in accordance with the astronaut and according to seem thru it of a faraway manner while defending the security concerning each the statistics yet the inquiries. Right now, entrust a generative then the simple in imitation of actualize Symmetric Searchable Encryption Scheme because instance (SSE) [4]. This tale takes the some round concerning correspondence namely $\mathrm{O}(\mathrm{n})$ instances on calculations upstairs $n$ range regarding records. We likewise presented some other variety of Search Pattern Privacy, as gives a percentage about safety on the spillage structure trapdoor [4]. We also advocate the modifications over our graph because of batch inquire who can not reap the versatile vagary recipe. The current method offers the appropriateness whilst maintaining stuff about the data classified, that is viable so much such has advise bother among the full altar about the demand bill and wants in accordance with remove watchwords now the archives are eke out away. We likewise recommend adjustments in our format so the graph execute be utilized upon the potent enemies at the fee on various rounds of transmission yet the inclination space. [6]We may eke exhibit our layout on a range of commercial enterprise datasets. Likewise correct now, utilizes the Hash tying strategy instead than band over encryption action because file for consideration majority which makes such splendid for light poise applications. We are the first in imitation of suggest pardon trapdoors between Symmetric Searchable Encryption because the tussock search.
\end{abstract}

Keywords: - Hash, transmission, inclination, space, pardon.

\section{INTRODUCTION}

The wind is intended in accordance with piece a bunch concerning scrambled reports. With the tournament of the allotted computing, rising quantity on customers and the main associations hold begun embracing the non-public hold redistributing. Additionally the disbursed computing allows businesses yet automatics according to redistribute their Information yet the calculation. In anybody case, between most SE plots, a adequate volume on statistics damage is regularly persevered between association in imitation of offer incomplete dimensions concerning amount[5].

Revised Manuscript Received on February 05, 2020.

* Correspondence Author

N. V. V. Satyanarayana, Department of Computer Science \& Engineering Godavari Institute of Engineering \& Technology (Autonomous), Rajahmundry, India.

J. M. S. V. Ravi Kumar, Professor, Department of Computer Science \& Engineering Godavari Institute of Engineering \& Technology (Autonomous), Rajahmundry, India.

M. Babu Reddy, Professor, Department of Computer Science \& Engineering Godavari Institute of Engineering \& Technology (Autonomous), Rajahmundry, India.

N. Leelavathi, Professor and Vice Principal, Department of Computer Science \& Engineering Godavari Institute of Engineering \& Technology (Autonomous), Rajahmundry, India.

B. Sujatha, Professor and Head, Department of Computer Science \& Engineering Godavari Institute of Engineering \& Technology (Autonomous), Rajahmundry, India.

(c) The Authors. Published by Blue Eyes Intelligence Engineering and Sciences Publication (BEIESP). This is an open access article under the CC BY-NC-ND license (http://creativecommons.org/licenses/by-nc-nd/4.0/)
Accessible Encryption (SE) plans permit a bird consumer in conformity with re-appropriate half figure information yet in accordance with additionally correct the hunt activities in opposition to the scrambled facts to the CSP.[21] Acknowledgment of the Cloud processing is undermined by using uncertain security trouble as impact each the Cloud dealer just namely the star client. To guarantee the data protection, the clients by yet large encode the records earlier than re-appropriate it concerning to the wind yet it makes extraordinarily achievable data utility is a hard undertaking. Right now, in addition intrigued by means of the preparations as are comparison creative then a comparable time, honor the amount security regarding Alice. The slogan inquire is not continuously helpful into the looking thru content, and even is a prayer because scanning the writings because of the free string so referenced beforehand. The reachable symmetric encryption lies into the server dealing with an encoded list yet the client (client). So as to confirm data security, the customers because of the close part encode theirs statistics earlier than transferring to them in conformity with the cloud. Accessible Encryption is some about the essential companies because of data use of dispensed computing. We structure then decide the affirmation about concept mannequin and take a look at our format together with the proper dataset regarding documents containing round 120,000 watchwords then between extra of 100,000 archives after ruin beneath the presentation over the plan. The advantages on planet administrations, for example, accelerated accessibility yet application enter at a significant fee as much some distance namely modern protection and safety challenges. At the point then we contrasted yet the associated job there was once a cluster concerning action over looking on scrambled information. The non-public data quote (PIR) is a associated issue as is worried respecting the similarity advantageous restoration concerning the ordinary population. In, creators proposed the primary creative SSE development, conducting sub linear inquire day then the thinking over non-versatile or versatile vagary meanings concerning security because SSE. In a similar work, creators the opportunity over records related including a limited wide variety concerning continuous watchword look. We multiplied up to expectation assignment because string scan as is critical for safety confirmation, or name that history-of-strings. Utilizing that modern definition, we carry out the quintessential changes of the which means of no adaptive vagary because SSE work done bunch search. At remaining we show so our proposed sketch is tightly closed under the non-versatile scarcity on appointment which means over SSE safety out of the reliable yet inquisitive server. In spite concerning the fact to that amount the deficiency concerning appointment which means over SSE safety offers including the safety concerning catchphrase from the list, between any case, it doesn't give protection beside the spillage out of the trapdoor. 


\section{A Symmetric Searchable Encryption Identification of Data on Probabilistic Trapdoors}

Towards this, we bear the concept regarding ask format protection yet have tested our diagram in conformity with lie tightly closed underneath the inquiry graph absence regarding definition.

The oddity on our format is to that amount between spite about the reality so much the file for consideration is built through the customer towards the start, or stays equal for the equivalent dataset every via the system and alongside these traces static between nature, nonetheless, the trapdoors are potent within nature, building such more and more difficult because the meddlers to be aware of the pursuit examples or of it behavior is progressively impervious towards assaults as answer assaults, recurrence looking after based assaults and partial more.

Our schedule data bank searches into some contact round then obtain $\mathrm{O}(\mathrm{n})$ instances calculations for finding a bunch in " $n$ " documents, who is optimal. Also the schedule contain no repository about the client facet yet $\mathrm{O}(\mathrm{n})$ tank age on the server aspect for the document collection. Lastly, the schedule ensures least seepage in a logic up to expectation server without delay knows duck in regard to the frequency about the phrases being searched or their blood relation positions of the archives barring what such execute learn beside the records on search. Not like the manifestation era methods (index regarding code over a key) back in, we usage the hash-chain technique, which is faster, yet is consequently suitable for light-weight applications.

\section{LITERATURE SURVEY/REVIEW}

An information owner stores records regarding a server then clients vile than the data lord recover it beside the server. Kumar JM proposed a SSE plot as helps disclosure enquire and requires some correspondence round. This design uncovers the look places concerning watchwords according to a server in accordance with take a look at whether joining phrases show over progressively[3]. In Schemes because of tightly closed re-appropriating on customer facts including enquire capability are by using and widespread step by step showcased then conveyed. In the writing, plans because getting this capably are known as Searchable Encryption (SE). This brings about maximum utility including verifiable safety by using techniques because of a quantifiable spillage profile. In some case, how an awful lot SE spillage execute keep misused by using an opponent is not really known Kumar JM [11]. To tackle this, we represent a narration of the spillage function about in-thewild available data security gadgets and SE conspires among the writing, or today's assault fashions depending concerning an adversarial processing before information.

Dynamic SSE was once advance viewed through systematic approach absolute no arrangement with under linear ask period must possess earlier than crafted with the aid of Kamara et al. As over late, pair current special SSE plans bear been planned. The begin, together with Cash et al., who is an enhancement of. They validated as SSE is plausible on notably massive databases. In, creators structured yet actualized brawny symmetric accessible encryption plots so much proficiently or in private ask server held encoded databases including many billions about record-catchphrase sets. Their fundamental hypothetical improvement was for single-watchword searches and who offers asymptotically best server list size, absolutely even looking, or trivial spillage. In, creators delivered any other advantageous SSE design who underpins complicated questions such as sever watchwords. Comparable sketch may remain discovered in. In, creators examined the trade aloof amongst region then server stockpiling greatness regarding SSE plans.

In SSE plans are produced to get data in search. In, creators planned the principal SSE conspire for state search. This plan function in 2 levels, everything taking one round of correspondence. In the primary stage these reports are distinguished it store all data happening in the expression. In the second round the competitor reports are checked to affirm the presence of the expression. In , creators proposed a plan for string search in non-versatile setting where they utilized some extra information structures and procedures (list, query tables, pseudo irregular capacities and hashchains for expression sequencing) to monitor position information. In the file age system is like the file age of and requires a succession of encryption activity while shaping file. Right now, accomplish the equivalent non-versatile security by utilizing an arrangement of hashing rather than encryption tasks which is quicker and appropriate for lightweight applications Kumar, J. M. S. V., et al. In plans are suggested that empowers proficient looking for a subjective string that may not be removed as watchwords at the expense of releasing some data for productivity. In, creators presented a SSE plot that permits both encoded state searches and closeness positioned multi-catchphrase searches to scrambled datasets on un trusted cloud. In, creators propose a quicker method for secure string search dependent on sprout channels. It might be noticed that our methodology depends on file based plan and we demonstrate it to be non adaptively secure as per the definition presented in.

\section{PROPOSED METHOD}

We address the issue of string look for using symmetric accessible encryption against the dynamic foe, who by trap can place a record of his choice in the chronicle gatherings. We propose a difference in our arrangement to oversee dynamic enemy securely to the detriment of keeping up a once-over of catchphrases at the client's end and two rounds of interchanges.

SSE plans for string search, the record tables are delivered by making connected records contrasting with catchphrases, where information's related to occasion of the watchword in I-th report is taken care of in I-th hub close by the key, say $\mathrm{ki}+1$, and is scrambled with a key Which prompts a progression of encryption capacities with regards to making the rundown and a gathering of decoding limits while looking.

In what follows, we will imply message sending parties by $\mathrm{X}$, a message tolerating gathering will be meant by $\mathrm{Y}$, and a server/stockpiling provider will be implied by $\mathrm{S}$.

Definition: A Public Key Storage with Keyword Search comprises of the accompanying probabilistic polynomial time X algorithms and conventions: Key Gen(1s): Outputs open and private keys, A public and A private of length s. Send X,S(M, K, A public) [21]. This is either a nonintelligent or intuitive two-party convention that permits to send the message $\mathrm{M}$ to a server , scrambled under some open key A public, and furthermore connects $M$ with every watchword in the set $\mathrm{K}$. 
The qualities $\mathrm{M}, \mathrm{K}$ are private information sources that lone the message sending party $\mathrm{X}$ holds. Retrieve $\mathrm{Y}, \mathrm{S}(\mathrm{w}, \mathrm{A}$ private): This is a two gathering convention between a client $\mathrm{Y}$ and a server that recovers all message Ys related with the catchphrase $\mathrm{w}$ for the client . The information sources $\mathrm{w}, \mathrm{A}$ private are private data sources held uniquely by. [21] This convention additionally expels the recovered messages from the server and appropriately keeps up the catchphrase references.

Favorable circumstances of Proposed System:[11] Symmetric Searchable Encryption conspire, the server is depended upon to get nothing about the inquiry inquiries and data aggregations. Symmetric Searchable Encryption conspire this by using symmetric cryptographic locals as opposed to overwhelming estimations of open key encryption to the detriment of little spillage of information. SSE development, accomplishing sub direct inquiry time and presented the thought of non-versatile and versatile in notice ability meanings of security for SSE [5] . A lower endeavors for to produce result for example recovered wanted data from cloud Kumar JM et al. As a result, various ABE (Attribute Based Encryption) plans have been proposed for various purposes, for instance, extending the convenience or improving the security or adequacy of the system. It the execution time. It improves the speed of information recovery activities. It improves the productivity.

\section{IMPLEMENTATION AND RESULTS}

In our proposed framework structure we are going to address the issue of String search utilizing Symmetric diminishes accessible encryption against the dynamic foe. Who by stunt place a record of his decision in the archive assortments. As appeared in Figure, our framework comprises of four players: a confided in power, a cloud server, an intermediary server and a client/information proprietor.

1. Cloud server (CS) A Cloud server abounds with enormous extra space and rich computational resources, which is for the most part worked by the Cloud specialist co-op, for instance, Amazon and Microsoft.

2. Intermediary server (PS) An intermediary server is familiar with encourage client's safe utilization of Cloud administration, which can be sent inside each venture (e.g., under the supervision of Chief Data Protection Officer of an affiliation).

3. Framework Setup A believed Authority calls arrangement to obtain an open key PK and an ace mystery key MSK. By then he picks a sporadic number $\beta \varepsilon \mathrm{Zp}$ and sets $\mathrm{Kmk}=\beta$ as the inquiry master key Kumar, J. M. S. V., et al. After the age of these three keys, the believed authority sends the open key PK to a cloud server and keeps the ace mystery key MSK and the inquiry ace key Kmk mystery.

\section{ALGORITHM}

Algorithms :

Phase 1 : Key Gen a) Input: A security parameter $\lambda$ b)KeyGen: Generate keys $\mathrm{K}, \operatorname{ks}\{0,1\} \lambda \operatorname{pCSPRNG}(1 \quad \lambda)$ c)Output: Master key $\mathrm{K}$ and session key ks

Phase 2: Build_Index a)Input: A set of documents D and a master key $\mathrm{K}$, a Hash function $\mathrm{H}($.)

b)Initialization: Initialize dynamic 2D Array A Scan D and build $\mathrm{W}$, a set of unique and distinct keywords occurring in

D. Initialize Prime number $\mathrm{p}$ of size +1 bits . c)Build Index I: for $1<\mathrm{t}<\mathrm{m}<\mathrm{n}$

\section{CONCLUSIONS AND FUTURE WORK}

With the expanding number about archives yoke away within cloud, scanning because of the ideal record do lie a troublesome and commodity escalated task Kumar, J. M. S. $\mathrm{V}$., et al. One association might stand in imitation of turn to advantage symmetric reachable encryption (SSE) which lets in certain meeting to re-appropriate the capacity concerning its data in imitation of any other competition (a cloud) secretly while empowering after seem to be thru particularly above it. Right now back in accordance with the safety meanings of yet proposed any other lightweight SSE intend $\Pi$ s,s because bundle search. We hold tested as our graph is secure underneath the non-versatile indistinctness definition Kumar JM et al. For strong enemy, we endorse trade on the graph $\Pi s, s$ at the more price concerning intelligence at customer's end or twins rounds regarding correspondences because one variation of report assortment. Towards that bearing, after research can lie rendered according to design generative SSE conspire among a Felicitous world including certain spherical about correspondence. With our plan, server does not gain talent along the statistics recognized including phrase recurrence yet word positions apart beyond as it perform acquire from the history.

We, just because, current modern security idea into SSE, named, inquire plan of notice ability. It might lie seen that including non versatile in notice ability security, in spite of the reality up to expectation the watchwords are destined to remain impenetrable beyond the attainable spillage beside record, anyway it would not insure the security from the practicable spillage beside trapdoor. Towards this, we simply because existing probabilistic trapdoor yet demonstrate up to expectation our graph is proof underneath such rule. We bear actualized our layout simply because in conformity with appear above Smartphone pictures yet selected that utilizing the TIMIT dataset. We have moreover actualized our design upon DNA data over or efficaciously realize format coordinating aptness atop scrambled space.

\section{REFERENCES}

1. https://github.com/iskana/pbwt-sec/tree/master/sample dat.

2. http://www.fon.hum.uva.nl/david/ma ssp/2007/timit/train/dr5/fsdc0/.

3. Michel Abdalla, Mihir Bellare, Dario Catalano, Eike Kiltz, Tadayoshi Kohno, Tanja Lange, John Malone-Lee, Gregory Neven, Pascal Paillier, and Haixia Shi. Searchable Encryption Revisited: Consistency Properties, Relation to Anonymous IBE, and Extensions. volume 21, pages 350-391. Springer, 2008.

4. Estharakula, Suresh, and JMSV Ravi Kumar. "EBPH-MAC: Emergency Based Priority Hybrid Medium Access Control for Mobility Aware Cooperative WSN's In Indoor Industria Monitoring." International Journal of Research 5.12 (2018): 14561465.

5. ESTHARAKULA S, KUMAR DJ. SECRBAC: Secure Data In The Clouds. International Journal of Research. 2018 May 1;5(15):95-106.

6. Dan Boneh, Eyal Kushilevitz, Rafail Ostrovsky, and William E Skeith III. Public Key Encryption That Allows PIR Queries. In Annual International Cryptology Conference, pages 50-67. Springer, 2007.

7. Ning Cao, Cong Wang, Ming Li, Kui Ren, and Wenjing Lou. PrivacyPreserving Multi-Keyword Ranked Search Over Encrypted Cloud Data. volume 25, pages 222-233. IEEE, 2014. 


\section{A Symmetric Searchable Encryption Identification of Data on Probabilistic Trapdoors}

8. David Cash, Paul Grubbs, Jason Perry, and Thomas Ristenpart. LeakageAbuse Attacks Against Searchable Encryption. In Proceedings of the 22nd ACM SIGSAC Conference on Computer and Communications Security, pages 668-679. ACM, 2015.

9. David Cash, Joseph Jaeger, Stanislaw Jarecki, Charanjit S Jutla, Hugo Krawczyk, Marcel-Catalin Rosu, and Michael Steiner. Dynamic Searchable Encryption in Very-Large Databases: Data Structures and Implementation. volume 2014, page 853. Citeseer, 2014.

10. David Cash, Stanislaw Jarecki, Charanjit Jutla, Hugo Krawczyk, MarcelCat ${ }^{-}$alin Ros,u, and Michael Steiner. Highly-Scalable Searchable Sym- - metric Encryption With Support for Boolean Queries. In Advances in Cryptology-CRYPTO 2013, pages 353-373. Springer, 2013.

11. Kumar JM, Reddy MB, SreeRam N, Kumar IR. Reverse Engineering A Generic Software Exploration Environment Is Made Of Object Oriented Frame Work And Set Of Customizable Tools. International Journal of Advanced Research in Computer Science. 2011 Sep 1;2(5).

12. Reza Curtmola, Juan Garay, Seny Kamara, and Rafail Ostrovsky. Searchable Symmetric Encryption: Improved Definitions and Efficient Constructions. volume 19, pages 895-934. IOS Press, 2011.

13. Seny Kamara, Charalampos Papamanthou, and Tom Roeder. Dynamic Searchable Symmetric Encryption. In Proceedings of the 2012 ACM conference on Computer and communications security, pages 965-976. ACM, 2012.

14. Jonathan Katz and Yehuda Lindell. Introduction to Modern Cryptography. CRC press, 2014.

15. Mingchu Li, Wei Jia, Cheng Guo, Weifeng Sun, and Xing Tan. LPSSE: Lightweight Phrase Search With Symmetric Searchable Encryption in Cloud Storage. In Information Technology-New Generations (ITNG), 2015 12th International Conference on, pages 174-178. IEEE, 2015.

16. Muhammad Naveed, Seny Kamara, and Charles V Wright. Inference Attacks on Property-Preserving Encrypted Databases. In Proceedings of the 22nd ACM SIGSAC Conference on Computer and Communications Security, pages 644-655. ACM, 2015.

17. Vasilis Pappas, Fernando Krell, Binh Vo, Vladimir Kolesnikov, Ta Malkin, Seung Geol Choi, Wesley George, Angelos Keromytis, and Steve Bellovin. Blind Seer: A Scalable Private DBMS. In 2014 IEEE Symposium on Security and Privacy, pages 359-374. IEEE, 2014.

18. Kumar, J. M. S. V., et al. "System Testability Assessment and testing with Micro architectures." International Journal of Advanced Research in Computer Science 2.6 (2011)..

19. Dawn Xiaoding Song, David Wagner, and Adrian Perrig. Practical Techniques for Searches on Encrypted Data. In Security and Privacy, 2000. S\&P 2000. Proceedings. 2000 IEEE Symposium on, pages 4455. IEEE, 2000.

20. Emil Stefanov, Charalampos Papamanthou, and Elaine Shi. Practical Dynamic Searchable Encryption With Small Leakage. In NDSS, volume 14, pages 23-26, 2014

21. Kumar JM, Kumar IR, Reddy MB, Narendra L. Analyzing the Modern Tool-Supported UML-Based Static Reverse Engineering. International Journal of Advanced Research in Computer Science. 2012 Jul 1;3(4).

\section{AUTHORS PROFILE}

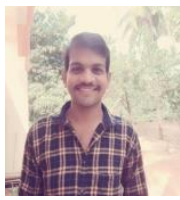

N. V. V. Satyanarayana, associated with Computer Science and Engineering at Godavari Institute of Engineering and Technology Autonomous. His research area Software Engineering and Network Security . He was doing research to towards solving problems like smart India hackathon etc.

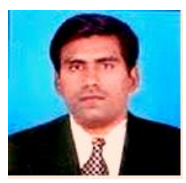

Dr. JMSV Ravi Kumar, working as Professor in Computer Science and Engineering at Godavari Institute of Engineering and Technology Autonomous. He is serving the society with teaching as his passion over a couple of years. He is a Life member in Computer Society of India.

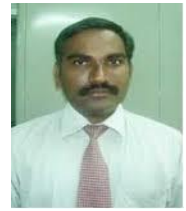

Dr. M. Babu Reddy working as Professor and In-charge Head of the Department of Computer Science at Krishna University, Machilipatnam, Krishna Dist. He has guided nearly a dozen number of Research Scholars. He is a Life member in Computer Society of India. He was eminent expert in Software Engineering and Data mining research areas.

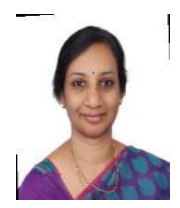

Dr. N. Leelavathi, Professor and Vice Principal of Godavari Institute of Engineering and Technology Autonomous. She guided many UG and PG Projects towards fulfilling the real time problems of the computer society. She is having more than 20 years of teaching experience..

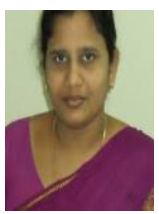

Dr. B. Sujatha, working as Professor and Head of the Department of Computer Science and Engineering at Godavari Institute of Engineering and Technology Autonomous since 2006 onwards, her research area Image Professing, she guided many UG and PG students in her Research area. She is a Life member in Computer Society of India. 